\title{
Protective Embolization of the Gastroduodenal Artery with a One-HydroCoil Technique in Radioembolization Procedures
}

\author{
R. López-Benítez $\cdot$ P. Hallscheidt $\cdot$ C. Kratochwil • \\ C. Ernst • L. Kara • O. Rusch • P. Vock • \\ J. Kettenbach
}

Received: 7 December 2011 / Accepted: 29 January 2012/Published online: 14 March 2012

(C) Springer Science+Business Media, LLC and the Cardiovascular and Interventional Radiological Society of Europe (CIRSE) 2012

\begin{abstract}
Purpose Protective occlusion of the gastroduodenal artery (GDA) is required to avoid severe adverse effects and complications in radioembolization procedures. Because of the expandable features of HydroCoils, our goal was to occlude the GDA with only one HydroCoil to provide particle reflux protection.

Methods Twenty-three subjects with unresectable liver tumors, who were scheduled for protective occlusion of the GDA before radioembolization therapy, were included. The primary end point was to achieve a proximal occlusion of the GDA with only one detachable HydroCoil. Evaluated parameters were duration of deployment, and early (during the intervention) and late (7-21 days) occlusion rates of GDA. Secondary end points included complete duration of the intervention, amount of contrast medium used, fluoroscopy rates, and adverse effects.

Results In all cases, the GDA was successfully occluded with only one HydroCoil. The selected diameter/length range was $4 / 10 \mathrm{~mm}$ in 2 patients, $4 / 15 \mathrm{~mm}$ in 6 patients, and $4 / 20 \mathrm{~mm}$ in 15 patients. HydroCoils were implanted, on average, $3.75 \mathrm{~mm}$ from the origin of the GDA (range 1.5-6.8 mm), with an average deployment time of 2:47 (median 2:42, range 2:30-3:07) min. In 21 (91\%) of 23 patients, a complete occlusion of the GDA was achieved during the first $30 \mathrm{~min}$ after the coil implantation; however, in all patients, a late occlusion of the GDA was present

R. López-Benítez ( $)$ · L. Kara · O. Rusch · P. Vock ·

J. Kettenbach

Inselspital Universitätsspital Bern, Freiburgstrasse 18,

3010 Bern, Switzerland

e-mail: Ruben.lopez@insel.ch

P. Hallscheidt - C. Kratochwil - C. Ernst

University of Heidelberg, Im Neuenheimer Feld 110,

69110 Heidelberg, Germany
\end{abstract}

after 6 to 29 days. No clinical or technical complications were reported.

Conclusion We demonstrated that occlusion of the GDA with a single HydroCoil is a safe procedure and successfully prevents extrahepatic embolization before radioembolization.

Keywords Clinical practice - Embolization .

Embolotherapy · Interventional oncology · Liver/hepatic · Radiation protection

\section{Introduction}

Selective internal radioembolization is a catheter-based, liver-directed therapy that involves the injection of micronsized embolic particles loaded with a radioisotope using percutaneous transarterial techniques. Unlike other locoregional therapies, preprocedure planning and meticulous mesenteric angiography are imperative in order to determine the safest and most effective treatment strategy [1]. Protective occlusion of the gastroduodenal artery (GDA) is frequently required before radioembolization because extrahepatic embolization (nontarget embolization) of yttrium-90 microspheres may lead to severe adverse effects and complications.

For this purpose, different materials, including microcoils, vascular plug devices, and more recently hydrogelcoated coils, have been used. The HydroCoil (MicroVention, Tustin, CA) is a coil system designed to improve coilpacking density; it comprises a layer of acrylic polymer surrounding a platinum metallic core that increases in thickness and diameter when in contact with liquid or blood.

Because of its unique expandable features, we proposed that the proximal occlusion of the GDA with only one detachable HydroCoil would be feasible and effective to 
provide protection against particle reflux in patients scheduled for selective internal radioembolization therapy (SIRT).

\section{Materials and Methods}

The present study was a single-center pilot study performed in a tertiary clinical institute. The study protocol was reviewed and approved by the ethics committee of our institution and conducted in accordance with good clinical practice (EU guideline EN ISO 14155).

\section{Study End Points}

The primary end point of this study was the proximal occlusion of the GDA using only one HydroCoil in patients who were undergoing SIRT. For this purpose, the HydroCoil implantation time duration, and the early (during the intervention) and late occlusion (7 to 21 days) rates of the GDA were evaluated.

As secondary end points, several technical variables were assessed, including complete duration of the intervention ( $\mathrm{min}$ ), contrast medium doses (ml), fluoroscopy rates, and local and systemic adverse effects.

\section{Inclusion Criteria}

Twenty-five subjects with unresectable primary and secondary liver tumors who were scheduled for radioembolization therapy were included. In all cases, the presence of the GDA with proven antegrade flow (GDA blood flow directed from the celiac trunk and not from the superior mesenteric artery) had to be demonstrated.

If necessary, other vessels (right gastric artery, gastroepiploic artery, small duodenal arcades, cystic, left gastric artery) were also embolized; however, these vessels were not assessed in this study. Patients were informed of the nature of the study, and written informed consent was obtained before the procedure.

\section{Exclusion Criteria}

Patients were excluded if they had any condition or had undergone any prior therapy that, in the opinion of the clinical investigator, would make the subject unsuitable for this study. Patients with GDA reversed flow (in these cases, protection of the GDA is usually not required [2]), were also excluded, as were patients with previous endovascular embolization by coils or surgical ligature/resection of the GDA, or with vascular anatomic variants, such as absence of the GDA.
Descriptive and Comparative Analysis

Our results were descriptively analyzed and compared to a similarly designed study performed by Dudeck et al. [3], who used a fibered interlock detachable coil system versus standard pushable coils.

Procedure

\section{Technique}

All procedures were performed with the patient under local anesthesia via 4F femoral access. Angiographic mapping of the mesenteric vessels, including selective angiograms from the celiac trunk and superior mesenteric artery, were performed with an S1 4F catheter (Cordis, Miami, FL). The angiographic information demonstrated the patient's vascular anatomy, the presence of anatomical variants, flow direction, and possible collateral vessels. For the coiling procedure, superselective catheterization of the GDA was performed with a $2.7 \mathrm{~F}$ microcatheter $(0.025$ inches inner diameter) (Terumo Europe, Heverlee, Belgium).

\section{HydroCoil Selection and Preparation}

Only 0.018-inch detachable HydroCoils were used in this study. The decision about which diameter and length of HydroCoil was suitable for each patient was performed indirectly from the French calculation method: knowing that $1 \mathrm{~F}$ equals $0.33 \mathrm{~mm}(4 \mathrm{~F}=\sim 1.2 \mathrm{~mm})$, the vessel diameter could be calculated on the basis of the selected catheter size. When possible, oversizing of the selected coil was avoided by fitting the maximum coil diameter to the vessel diameter if this was not feasible, a minimal oversizing to the next available diameter of HydroCoil was accepted (i.e., a vessel of $3.9 \mathrm{~mm}$ was embolized with a 4.0-mm HydroCoil rather than a $5.0 \mathrm{~mm}$ HydroCoil). All vessel measurements were performed by angiography at the origin of the GDA (Fig. 1).

All detachable HydroCoils and the electromechanical delivery device were tested before implantation according to the manufacturer's guidelines. To ensure optimal curling, the HydroCoil was briefly immersed (5 s) in sterile water at $70^{\circ} \mathrm{C}$.

\section{HydroCoil Implantation Zone}

The HydroCoil implantation was performed only in the most proximal segment of the GDA, where the first small pancreatic and duodenal branches arise. If the position of the HydroCoil was not satisfactory, repositioning was performed. Only when the desired position was reached was the HydroCoil detached. Angiographic control (hand 


\begin{tabular}{|c|c|c|}
\hline \multicolumn{3}{|c|}{ A } \\
\multicolumn{3}{|c|}{ French Catheter Scale } \\
\multicolumn{3}{|c|}{} \\
\hline *sizes are Outside Diameter \\
\hline French & INCH & MM \\
\hline 3 & 0.039 & 1 \\
\hline 4 & 0.053 & 1.35 \\
\hline 5 & 0.066 & 1.67 \\
\hline 6 & 0.079 & 2 \\
\hline 7 & 0.092 & 2.3 \\
\hline 8 & 0.105 & 2.7 \\
\hline 9 & 0.118 & 3 \\
\hline
\end{tabular}

Fig. 1 French catheter scale. Indirect calculation of GDA diameter using the outer diameter of the Sidewinder catheter as a reference diameter. A 4F catheter with a well-known diameter of $1.35 \mathrm{~mm}$ correlates with an approximate GDA diameter of 2.70 to $3 \mathrm{~mm}$. Only

injection of $2.5 \mathrm{ml}$ contrast medium at $1 \mathrm{ml} / \mathrm{s}$ flow) was performed immediately after the deployment (time 0 ) and every 5 min sequentially until complete vascular occlusion was observed, during a maximal period of $30 \mathrm{~min}$ (Fig. 2).

Median-term angiograms were performed in all patients 7 to 29 days after coiling just before at the SIRT procedure.

\section{HydroCoil Deployment Time and Time to Occlusion}

The HydroCoil deployment time was defined as the time from when the sterile package of the HydroCoil was opened until the HydroCoil was completely detached within the GDA. This included the following steps: opening of the sterile package; short immersion of the HydroCoil in a water bath at $70^{\circ} \mathrm{C}$ saline water; insertion of the HydroCoil within the microcatheter; positioning of the HydroCoil within the vessel; and detachment from the attached deployment wire. The time for occlusion was

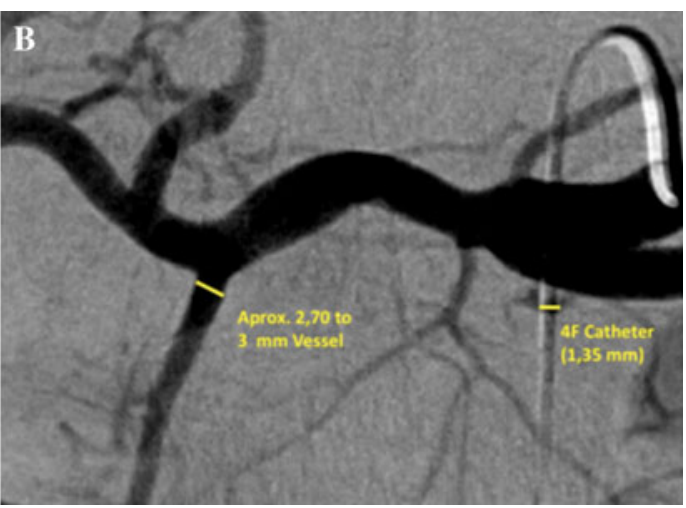

slight oversizing of the selected coil was being performed to avoid distal coil migration (i.e., a vessel approximately $3 \mathrm{~mm}$ in diameter would be embolized by a 4-mm-diameter HydroCoil)

defined as the period between HydroCoil detachment until angiographic evidence of complete proximal occlusion of the GDA, measured in time intervals of $5 \mathrm{~min}$.

\section{Vascular Effects after HydroCoil Implantation}

Angiographically significant flow reduction was defined as the moment when vascular stasis was evident, but there was not yet complete occlusion of the GDA (Fig. 3).

\section{Results}

Patient Demographics

From the 25 patients who underwent protective GDA embolization before SIRT, the planned SIRT was canceled in 2 patients $(8 \%)$ as a result of progressively impaired liver
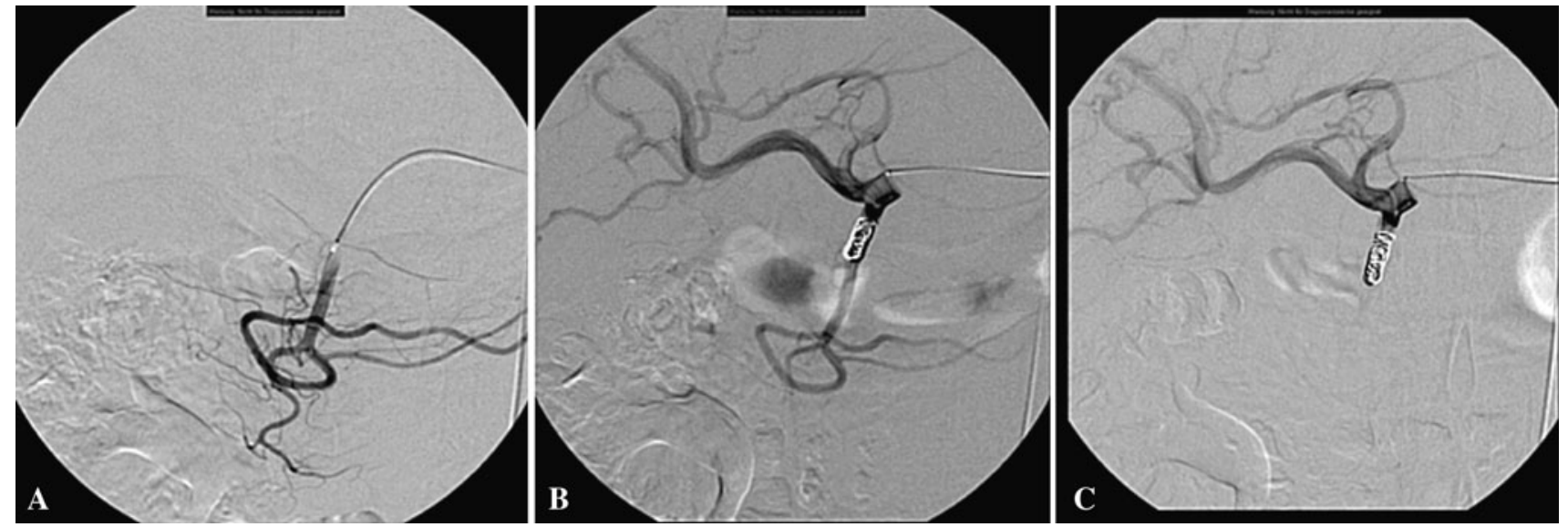

Fig. 2 Coiling phases of GDA. A Localization of the appropriate coiling segment. B Angiogram performed 5 min after HydroCoil implantation still shows flow; however, a flow reduction is already observed. C Angiogram performed 20 min after HydroCoil implantation demonstrates complete occlusion of the GDA 


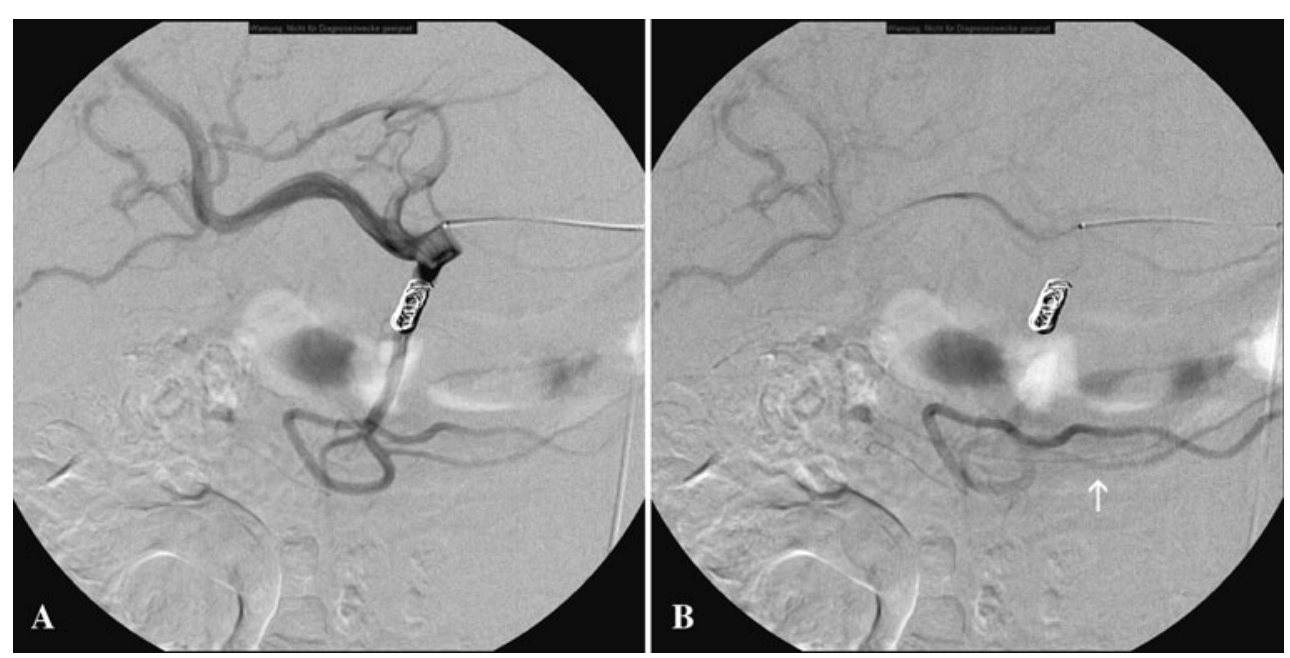

Fig. 3 Angiographically significant flow reduction. A Angiogram $1 \mathrm{~min}$ after device implantation. Intrahepatic vessels, as well the GDA and the gastroepiploic artery, are equally filled with contrast. B Angiogram performed $10 \mathrm{~min}$ after HydroCoil deployment clearly

function parameters; thus, angiographic control of the GDA was not possible. Therefore, the study sample included 23 patients (15 men and 8 women) with a median age of 56.2 years who had a liver or liver metastases due to the following: pancreatic neuroendocrine tumor $(n=8)$, colorectal carcinoma $(n=7)$, hepatocellular carcinoma $(n=4)$, breast cancer $(n=3)$, and malignant melanoma $(n=1)$. The median nominal diameter of the GDA before the HydroCoil implantation was 3.58 (range 3.10-4.60) $\mathrm{mm}$.

\section{HydroCoil Placement}

According to our study design, only one HydroCoil was implanted successfully in all cases. The range of selected diameter/length of the HydroCoils was as follows: $4 / 10 \mathrm{~mm}$ in 2 patients; $4 / 15 \mathrm{~mm}$ in 6 patients; and $4 / 20 \mathrm{~mm}$ in 15 patients. On average, the HydroCoils were implanted as close as 3.75 (range 1.5-6.8) $\mathrm{mm}$ from the origin of the GDA. The mean HydroCoil deployment time was 2:47 (median 2:42, range 2:30-3:07) min. An angiographically significant flow reduction was observed in all patients between 5 and 10 min before complete vascular occlusion was reached.

The desired embolization effect (time for occlusion), demonstrated by GDA occlusion, was observed as follows: two complete occlusions after $5 \mathrm{~min}$; four complete occlusions after $10 \mathrm{~min}$; six complete occlusions after 15 and $20 \mathrm{~min}$, respectively; two complete occlusions after $25 \mathrm{~min}$; and one complete occlusion after $30 \mathrm{~min}$. Thus, in 21 cases $(91.3 \%)$, a complete occlusion occurred during the first 30 min after GDA embolization (early occlusion), shows almost complete washout of the contrast medium in the intrahepatic arteries, whereas stasis of contrast-enhanced blood flow within the gastroepiploic artery (white arrow) is still visible

whereas in 2 cases $(8.7 \%)$, a complete occlusion of the GDA was not visible.

However, in all patients, a late occlusion was present after 6 to 29 (median 19) days when the late angiographic follow-up was performed (late occlusion rate $100 \%$, late recanalization rate $0 \%$ ). No clinical or technical complications related to the HydroCoil implantation (i.e., migration, dissection) were reported.

\section{General Procedure Results}

The mean complete duration of the procedures was 43:07 (range 22-110) $\mathrm{min}$. The total dose of contrast medium applied for the complete examination was 110 (range 90-135) $\mathrm{ml}$. In each case, two initial series with $35 \mathrm{ml}$ of contrast medium were performed $(35 \mathrm{ml}$ at a $6 \mathrm{ml} / \mathrm{s} \mathrm{rate})$ from the celiac trunk and the superior mesenteric artery. The other series were performed to determine the position of the microcatheter within the GDA, as well as to determine the effects of the occlusion after the HydroCoil embolization $(8 \mathrm{ml}$ at $1.5 \mathrm{ml} / \mathrm{s}$ rate). Additional series to occlude other vessels were performed in some cases, but these vessels were not considered part of our study. The median duration of fluoroscopy was 11:41 min for the whole procedure (range 6:27-17:53 min), with a mean time of 11:43 min.

\section{Adverse Effects}

There were no thromboembolic complications after GDA HydroCoil embolization. Recognizable clots, distal 
organ infarcts, or silent infarcts were not detected on postprocedure computed tomographic follow-up or diffusion-weighted magnetic resonance imaging.

None of the HydroCoil parts protruded (loop protrusion) from the GDA into other vessels (hepatic artery), and no distal migration was observed. No early liberation of detachable coils (within the microcatheter) or malfunction of the electromechanical delivery device were detected. None of the patients experienced allergic reactions related to hydrogel components or postembolization syndrome.

\section{Discussion}

We postulated that it would be possible to precisely occlude the proximal segment of the GDA to protect dependent territories, including the stomach, the duodenum, the pancreas, and the colon, from severe adverse effects as a result of the radioembolization procedures (radioembolic-related damage). However, the proximal occlusion technique of the GDA is not indicated for treating bleeding sources that arise from the GDA. In these cases, selective occlusion of the bleeding source should be attempted, or otherwise a complete GDA occlusion is mandatory.

Dudeck et al. [3] have already demonstrated that the use of the fibered interlock detachable coils for occlusion of the GDA in patients scheduled for SIRT was safe and effective, as well as associated with a shorter procedure time compared to the standard pushable fiber coils. In their study, a mean of $6.2 \pm 1.8$ standard coils were used to embolize the GDA, whereas a mean of $1.3 \pm 0.9$ interlock detachable coils were required. In our study, we further reduced that number to only one detachable HydroCoil necessary to achieve a satisfactory embolization result (Table 1).

HydroCoils are recently developed embolic (coil and microcoil) devices with a layer of hydrogel polymer surrounding a platinum metallic core that swells in blood [4]. The hydrogel polymer facilitates the expansion of the effective coil diameter up to several times its original diameter, thus not relying on the thrombogenicity of the blood alone, as the coil swelling results in reliable vessel occlusion without the need for distal embolization, as our study demonstrated [5,6]. This particular feature can also be helpful in patients who have an impaired coagulation profile when other embolization materials would need longer times to induce a vascular thrombosis or would need more coils [4].

To our knowledge, the application of HydroCoils used for protective coiling of the mesenteric vessels, particularly the GDA in patients who are undergoing SIRT, has not yet been assessed. Protective targeted embolization of the GDA is performed before the therapeutic procedure in order to confine the radioactive material to the liver [3, 7].

The precision of the HydroCoil deployment only a few millimeters away from the common hepatic artery (defined in our study as the neck, a distance that was a median of $3.7 \mathrm{~mm}$ ) demonstrates optimal material manageability. Repositioning the HydroCoils in cases of suboptimal delivery did not seem to be a problem unless the electromechanical mechanism had not been activated. Withdrawal of the HydroCoil, still attached to the deployment wire, is possible up to $5 \mathrm{~min}$ before the swelling, lubricated polymer coating prevents further repositioning through the microcatheter. This time span would allow repositioning of a misplaced microcatheter and reinsertion of the HydroCoil using the insertion sheath.

For protective embolization, it is essential to consider that small pancreatic and duodenal branch vessels may arise from the first millimeters of the GDA [8], also referred to as vessels. In radioembolization, precise placement of the embolization device in this segment is crucial to avoid radiogenic-related complications such as duodenitis or pancreatitis $[9,10]$. This is a valuable feature that other devices with a conical shape (i.e., the Amplatzer Vascular Plug IV) do not offer [11].

In our study, there were no observed cases of HydroCoil distal migrations, either during the early postimplantation phase or when the late angiographic control was performed.

Similar to other series that studied the performance of HydroCoils in cranial aneurysm embolization, the results of our study demonstrated that this material is an effective
Table 1 Comparison between three different embolization materials

\begin{tabular}{llll}
\hline Characteristic & $\begin{array}{l}\text { Pushable coils } \\
(n=25)\end{array}$ & $\begin{array}{l}\text { Fibered interlock detachable } \\
\text { coil }(n=25)\end{array}$ & $\begin{array}{l}\text { HydroCoil } \\
(n=23)\end{array}$ \\
\hline Embolization time (min) & $14: 32$ & $2: 15$ & $2: 47$ \\
Occlusion time (min) & $17: 18$ & $11: 19$ & $13: 02$ \\
Late recanalization (\%) & 4.3 & None & None \\
Mean number of devices & 6 & 1.3 & 1 \\
Cases requiring only one embolization & 0 & 83 & 100 \\
$\quad$ device according to study design $(\%)$ & & 4.3 & None \\
Migrations $(\%)$ & 4.3 & &
\end{tabular}


embolization tool in short- and long-term embolization periods (up to 29 days) [12-14].

In conclusion, we demonstrated the successful use of a single HydroCoil device for protective embolization of the GDA, with complete early-term vascular occlusion rates (during the first $30 \mathrm{~min}$ after embolization) in up to $91.3 \%$ of the cases, and definitively in all the cases in median-term vascular occlusion (up to 29 days). As demonstrated in our study, this material is also a suitable alternative that prevents extrahepatic embolization in radioembolization procedures.

Acknowledgments We are grateful for support by the LudwigBoltzmann-Institute of Vienna; and we are grateful to Mary McAllister, Johns Hopkins University Hospital, Baltimore, MD, for her assistance.

Conflict of interest The authors declare that they have no conflict of interest.

\section{References}

1. Bilbao JI, Garrastachu P, Herraiz MJ et al (2010) Safety and efficacy assessment of flow redistribution by occlusion of intrahepatic vessels prior to radioembolization in the treatment of liver tumors. Cardiovasc Intervent Radiol 33:523-531

2. Daghir AA, Gungor H, Haydar AA et al (2011) Embolisation of the gastroduodenal artery is not necessary in the presence of reversed flow before yttrium-90 radioembolisation. Cardiovasc Intervent Radiol. doi:10.1007/s00270-011-0208-9

3. Dudeck O, Bulla K, Wieners G et al (2011) Embolization of the gastroduodenal artery before selective internal radiotherapy: a prospectively randomized trial comparing standard pushable coils with fibered interlock detachable coils. Cardiovasc Intervent Radiol 34:74-80

4. Killer M, Arthur AS, Barr JD et al (2010) Histomorphology of thrombus organization, neointima formation, and foreign body response in retrieved human aneurysms treated with hydrocoil devices. J Biomed Mater Res B Appl Biomater 94:486-492

5. Guimaraes M, Uflacker R, Garretson JS et al (2011) Angiographic and histologic comparison of injectable, expansile hydrogel embolic and pushable AZUR embolic devices in porcine arteries. J Vasc Interv Radiol 22:1619-1624

6. Constant MJ, Keeley EM, Cruise GM (2009) Preparation, characterization, and evaluation of radiopaque hydrogel filaments for endovascular embolization. J Biomed Mater Res B Appl Biomater 89:306-313

7. Kennedy A, Coldwell D, Sangro B et al (2012) Integrating radioembolization $\left({ }^{90} \mathrm{Y}\right.$ microspheres $)$ into current treatment options for liver tumors: introduction to the international working group report. Am J Clin Oncol 35:81-90

8. Silveira LA, Silveira FB, Fazan VP (2009) Arterial diameter of the celiac trunk and its branches. Anatomical study. Acta Cir Bras 24:43-47

9. Ogawa F, Mino-Kenudson M, Shimizu M et al (2008) Gastroduodenitis associated with yttrium 90-microsphere selective internal radiation: an iatrogenic complication in need of recognition. Arch Pathol Lab Med 132:1734-1738

10. Sjoquist KM, Goldstein D, Bester L (2010) A serious complication of selected internal radiation therapy: case report and literature review. Oncologist 15:830-835

11. Pech M, Kraetsch A, Wieners G et al (2009) Embolization of the gastroduodenal artery before selective internal radiotherapy: a prospectively randomized trial comparing platinum-fibered microcoils with the Amplatzer Vascular Plug II. Cardiovasc Intervent Radiol 32:455-461

12. Deshaies EM, Adamo MA, Boulos AS (2007) A prospective single-center analysis of the safety and efficacy of the hydrocoil embolization system for the treatment of intracranial aneurysms. J Neurosurg 106:226-233

13. Gaba RC, Ansari SA, Roy SS et al (2006) Embolization of intracranial aneurysms with hydrogel-coated coils versus inert platinum coils: effects on packing density, coil length and quantity, procedure performance, cost, length of hospital stay, and durability of therapy. Stroke 37:1443-1450

14. Gunnarsson T, Tong FC, Klurfan P et al (2009) Angiographic and clinical outcomes in 200 consecutive patients with cerebral aneurysm treated with hydrogel-coated coils. AJNR Am J Neuroradiol 30:1657-1664 